\title{
Addressing Early Life Sensitivity Using Physiologically Based Pharmacokinetic Modeling and In Vitro to In Vivo Extrapolation
}

\author{
Miyoung Yoon and Harvey J. Clewell, III \\ The Hamner Institutes for Health Sciences
}

(Received December 1, 2015; Revised December 24, 2015; Accepted January 5, 2016)

\begin{abstract}
Physiologically based pharmacokinetic (PBPK) modeling can provide an effective way to utilize in vitro and in silico based information in modern risk assessment for children and other potentially sensitive populations. In this review, we describe the process of in vitro to in vivo extrapolation (IVIVE) to develop PBPK models for a chemical in different ages in order to predict the target tissue exposure at the age of concern in humans. We present our on-going studies on pyrethroids as a proof of concept to guide the readers through the IVIVE steps using the metabolism data collected either from age-specific liver donors or expressed enzymes in conjunction with enzyme ontogeny information to provide age-appropriate metabolism parameters in the PBPK model in the rat and human, respectively. The approach we present here is readily applicable to not just to other pyrethroids, but also to other environmental chemicals and drugs. Establishment of an in vitro and in silico-based evaluation strategy in conjunction with relevant exposure information in humans is of great importance in risk assessment for potentially vulnerable populations like early ages where the necessary information for decision making is limited.
\end{abstract}

Key words: Early life sensitivity, PBPK, IVIVE, Ontogeny

\section{INTRODUCTION}

A great deal of effort has been expended to promote the use of in vitro and in silico approaches in chemical safety assessment since the National Research Council (NRC) report on Toxicity Testing in the $21^{\text {st }}$ Century (1-3). The emphasis in the NRC report is on developing mode-ofaction based testing strategies using modern biology tools such as advanced cellular and molecular biology techniques to identify key cellular toxicity pathways and the molecular mechanisms by which chemicals perturb them, in order to predict adverse outcomes in humans. In this regard, application of the adverse outcome pathway (AOP) framework to human safety assessment has gained significant attention over the past few years. The AOP framework is analogous to the mode-of-action framework, except that it begins at a molecular initiating event, which is defined as the interaction of a chemical with a specific molecular tar-

Correspondence to: Miyoung Yoon, The Hamner Institutes for Health Sciences, ScitoVation, 6 Davis Drive, RTP, NC 27709 E-mail: myoon@scitovation.com

This is an Open-Access article distributed under the terms of the Creative Commons Attribution Non-Commercial License (http:// creativecommons.org/licenses/by-nc/3.0) which permits unrestricted non-commercial use, distribution, and reproduction in any medium, provided the original work is properly cited. get (4). The rest of the AOP consists of chemical agnostic response-response relationships. To apply this framework to chemical safety assessment, an internal exposure in an appropriate target tissue needs to be defined first in order to provide the concentration-effect relationship of a chemical for the key molecular initiating event. Then, the target tissue exposure can be translated to human daily exposure for conducting safety or risk assessment based on the AOP framework. Physiologically based pharmacokinetic (PBPK) modeling is an effective translation tool in this context for extrapolating from the internal exposure at the target to the equivalent exposure condition of interest, a process known as reverse dosimetry $(5,6)$. In addition, it provides a means to address sensitive populations, including early age humans, based on in vitro and in silico information.

\section{PREDICTING THE AGE-SPECIFIC TARGET TISSUE EXPOSURE USING IN VITRO AND IN SILICO APPROACHES TO SUPPORT RISK ASSESSMENT}

Rapidly changing physiology and biochemical processes during development and growth are expected to affect chemical kinetics in the growing body (7). The resulting differences in target tissue exposure (pharmacokinetics) may lead to age-associated sensitivity to chemical effects instead of or in addition to the true difference in tissue sensitivity 
(pharmacodynamics) to the chemical exposure. Thus, agedependent changes in pharmacokinetics have to be taken into account to properly evaluate a potential difference in sensitivity to the effects of a chemical in different life stages. Evaluating the impact of age-dependent pharmacokinetics on chemical sensitivity in humans is, however, challenging due to experimental and ethical reasons. Using animal studies to infer human early life sensitivity has several limitations, mainly due to the well-known species differences in physiological and biochemical changes during maturation, especially metabolism. Both the types of metabolism enzymes and their ontogeny patterns differ between species (8). There are also other age-dependent physiological factors that affect the clearance of compounds during early life, such as the blood flow to the liver, intestinal absorption, renal clearance, etc. (9). Reliance on animal studies to infer human infant and child sensitivity cannot account for these factors.

Here we proposed an alternative approach to improve risk assessment for early ages using PBPK modeling in conjunction with in vitro metabolism studies. The advantage of using a physiologically based modeling approach is that it provides a platform to incorporate and integrate diverse and independent information on the key determinants of target tissue exposure across life stages (Fig. 1). The parameters in a PBPK model can be categorized into four types in general: exposure, physiological, partitioning, and metabolism (10). The exposure parameters are determined solely by the characteristics of the exposures, such as route and rate. It is important to incorporate age-appropriate exposure information when predicting target tissue exposure in specific ages of concern. This is provided through another type of modeling, namely exposure simulation modeling performed with software platforms like the Cumulative and Aggregate Risk Evaluation System (CARES) or the Stochastic Human Exposure and Dose Simulation (SHEDS) model. The outputs from the exposure model can be used as inputs in the PBPK models $(11,12)$. The physiological parameters are available from the literature (13). Age-dependent changes in physiol-

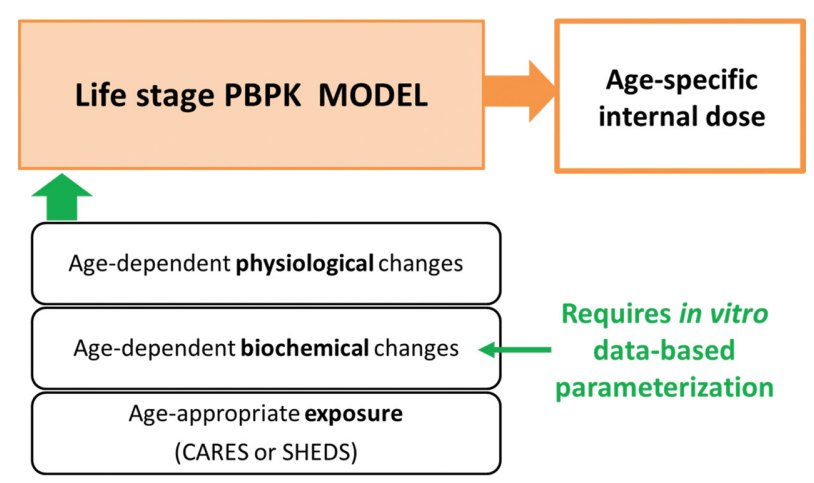

Fig. 1. Integrating the key determinants of early life dosimetry using PBPK modeling. ogy include increases in body weight, tissue weight, and tissue blood flows. Several reviews are available for these parameters in growing humans $(9,14,15)$. These types of parameters are not chemical-specific, and the values used in the evaluation of an untested compound would be the same as those used for well-characterized case compounds. Partitioning and kinetic parameters, however, are chemical-specific and need to be estimated for untested compounds (10). Age-dependent changes in biochemical processes include metabolism, partitioning, and plasma protein binding (9). Obtaining these parameters is the major challenge in developing PBPK models in human infants and children. In our approach, in vitro studies are used to provide age-appropriate metabolism parameters to a life stage PBPK modeling platform. This approach has gained strong support for predicting in vivo metabolic clearance on the basis of in vitro data using biologically based scaling processes $(6,16)$. By using age-appropriate exposure, physiological- and biochemical parameter values, PBPK models are well equipped to predict age-specific tissue internal exposure at the target tissue.

\section{USE OF IN VITRO METABOLISM DATA FOR EARLY LIFE PBPK MODEL DEVELOPMENT}

With the recent advances and improvements in in vitro assays, in vitro-derived metabolic constants have been increasingly used in PBPK models through IVIVE (6). The bottom-up approach, i.e., an in vitro data-based development of PBPK models, has been widely accepted and has become very popular for drug development in the pharmaceutical industry because of the growing availability of generic PBPK modeling software, such as Simcyp, in the past decade. Such models accept user-collected in vitro metabolism data such as Vmax and $\mathrm{Km}$ or intrinsic clearance values collected from subcellular fractions, primary hepatocytes, or expressed enzymes etc. and predict not only kinetics for a single drug, but also for drug-drug interactions (17-19). The bottom-up PBPK modeling approach actually started in environmental chemical risk assessment even before the popularity of Simcyp and other generic PBPK software for pharmaceutical compounds. It was initially applied for volatile organic chemicals and then later was extended to other chemicals including pesticides $(6,12,20-24)$. In general, the rationale for IVIVE is that the capacity of metabolism (e.g., Vmax) can be related between in vitro and in vivo by considering the total amount of enzyme present in each system. The affinity of metabolism (e.g., $\mathrm{Km}$ ) can be related by considering free, i.e., available, substrate concentration for enzyme reaction in each system (25). Therefore, in vitro-measured metabolic constants can be 'scaled up' to respective metabolism parameters in vivo in the PBPK model by relating enzyme content in vitro, e.g., Vmax per mg protein in vitro, to that in vivo, 
e.g., Vmax per g liver in vivo. There are several different in vitro systems available for metabolism studies and biological scaling factors (or steps) will vary depending on which in vitro system is used (6).

One of the major application areas of the bottom-up PBPK modeling approach is prediction of drug pharmacokinetics in pediatrics, clearly showing the value of using an in vitro-based approach to overcome data limitations in early life stages. The performance of IVIVE-PBPK models for eleven drugs showed that prediction of in vivo clearance of drugs in neonates, infants and children is possible based on in vitro data (26). This approach has shown its applicability to early life PBPK modeling for environmental chemicals as well $(12,22,27,28)$. The source-to-outcome model of chlorpyrifos is a good example of an IVIVEPBPK model that is accepted by the US EPA $(12,22)$. Price and colleagues (12) demonstrated the ability of this IVIVEPBPK model to predict age-specific target tissue exposure to chlorpyrifos and its active metabolite, chlorpyrifos oxon, concentrations in the brain at age 3 in comparison to age 30 . Furthermore, this model allowed prediction of the associated pharmacodynamic responses under age-appropriate exposure conditions for chlorpyrifos. A recent study on the integration of dosimetry and population variability into high-throughput screening assays for selected US EPA ToxCast chemicals is another good example of IVIVE-PBPK modeling applied to address potential sensitive populations including early ages in risk assessment (28). In this study, chemical specific adjustment factors (CASFs) for susceptible populations were estimated using the steady-state blood concentration in each population estimated based on IVIVE.

The availability of in vivo human data for pediatric populations for environmental chemicals is necessarily limited due to obvious ethical reasons. As described earlier, the data from neonatal animals would not be an appropriate alternative because of the well-known species differences in xenobiotic metabolism and maturation characteristics (8). The IVIVEbased parameterization is a promising alternative to obtain biochemical parameters for early ages instead of conducting in vivo studies using neonatal animals or collecting child data for optimizing parameters, which has been the major obstacle in expanding the utility of PBPK modeling to early age risk assessment for environmental chemicals.

\section{EARLY LIFE PBPK MODELING OF PYRETHROIDS - A CASE STUDY}

A proof of concept study in rats. Although it is an already accepted concept and has become a common practice in drug PBPK models for pediatrics $(26,29,30)$, to validate this approach for a specific class of environmental chemicals, e.g., pyrethroids in this case, a case study has been being conducted. First, a PBPK model for growing rats for a pyrethroid is developed to show proof-of-con-

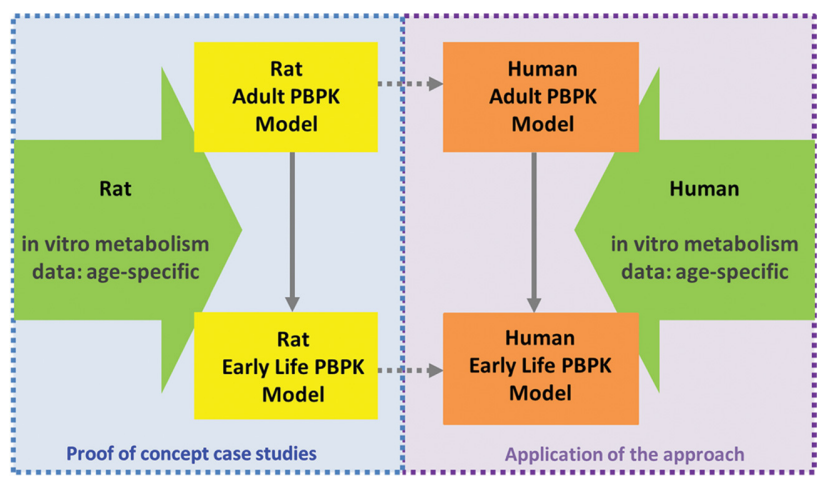

Fig. 2. IVIVE-PBPK modeling to predict early life dosimetry.

cept. Then the next step is to apply this method to develop an age-specific PBPK model for the pyrethroid in humans (Fig. 2). Deltamethrin was used as a case pyrethroid in our study. It serves as a good example for this purpose considering the observed early life sensitivity to high doses of this pyrethroid in rats (31). The increased lethality in neonatal rats has been regarded to be due to immature metabolizing enzymes resulting in higher target tissue concentrations of deltamethrin in young rats than in adults $(32,33)$. Pyrethroids including deltamethrin are metabolized by several cytochrome P450 (CYP) enzymes and carboxylesterase (CES) enzymes in the liver both for humans and rats and by carboxyesterases in the plasma only for rats $(34,35)$. The relative contribution between oxidative and hydrolytic metabolism pathways varies depending on the pyrethroid and substantial species differences also exist (34-37). In addition, maturation profiles, or ontogeny of, CYP and CES enzymes in humans are different compared to rats $(8,38,39)$. Given the experimental evidence, it is likely that the differential ontogeny of these enzymes is a key driver for the agerelated sensitivity observed in rats after the high dose of deltamethrin (31).

PBPK models for growing rats have been refined for deltamethrin using in vitro metabolism data (40) based on the previously published PBPK models for this pyrethroid $(41,42)$. In vitro metabolic constants collected from hepatic microsomes (32) and cytosol (43) prepared from postnatal day (PND) 10, 21, 40 and 90 rats were used for IVIVE of oxidative metabolism of deltamethrin by CYPs and hydrolysis by CESs. Plasma samples were collected from these rats and used to determine age-specific metabolic constants for hydrolysis in plasma for this pyrethroid by CESs. The current model therefore, describes the age-dependent changes in deltamethrin metabolism as a function of developmental changes in pyrethroid metabolism both by CYPs and CESs. Other physiological and biochemical processes occurring during development in rats were also included in this refined PBPK model for deltamethrin. This model performed satisfactorily in predicting in vivo kinetics of deltamethrin in a growing body of rats when we used in vitro- 
derived metabolism parameters in the model (40). Our results show that the observed age-related sensitivity in rats is indeed largely attributable to the limited metabolic capacity in young rats, e.g., PND10 and PND21, when they were treated with a high dose of deltamethrn (31). It should be noted that this case study in rats is to demonstrate the validity of IVIVE methodology of using age-specific metabolism data collected in vitro in estimating the total hepatic metabolic clearance of this pyrethroid in vivo. The utility of the model-estimated deltamethrin concentrations in brain or plasma in rats is to compare to those from in vivo PK studies performed in rats, not to extrapolate those to respective concentrations in humans.

Application of the IVIVE approach to humans. The human PBPK model for deltamethrin in different ages is currently under development using the IVIVE approach evaluated in the rat described above (40). We present the preliminary modeling results here to show the concept and workflow, although the final form of the model may be slightly different from the current version of the model. For humans, a different in vitro system is selected for metabolism. Instead of using subcellular fractions prepared from age-specific liver donors, expressed enzymes are used to provide metabolic constants for each age. Then the metabolism profile can be reconstituted to represent the whole liver across ages when combined with enzyme ontogeny data (6). The approach used for rat model development was not applicable for human model development, because age-specific pediatric liver samples would be required to obtain subcellular fractions. The availability of such liver samples is undoubtedly limited for humans. In addition, the quality of those samples in terms of metabolic activity may not be guaranteed. Even if a small number of pediatric liver samples were available, this method presents another issue, a large variability among individuals in humans. This is especially an issue in metabolizing enzyme expression in early ages (38) and cannot be adequately represented with a small number of tissue donors, which would potentially lead to a biased estimate of in vivo hepatic clearance. By using expressed enzymes, it is possible to provide the intrinsic metabolic capacity and affinity of each enzyme for a given compound. The necessary information on human variability in metabolism can be covered by population variability in enzyme expression levels.

The ideal work flow is as follows. First, one determines the metabolic constants for each of the CYP and CES enzymes that are involved in metabolism of a given pyrethroid. Then the intrinsic metabolic clearance (or Vmax and $\mathrm{Km}$ ) of each active enzyme is integrated in the PBPK model to obtain a total intrinsic metabolic clearance for the pyrethroid in the whole liver. During this IVIVE, the relative abundance of each enzyme in the liver is taken into account. This approach has been demonstrated for several drugs (26) and environmental chemicals (28). For preliminary simulations presented in our on-going study (40), the average adult intrinsic clearance in vivo ( $\mathrm{Clint}_{\text {in vivo }}$ ) was estimated using the data from subcellular fractions instead of expressed enzymes (43). As the major contributor to deltamethrin metabolism in both microsomal and cytosolic fractions in the liver is known to be CES-1 (39), the estimated Clint $_{\text {in vivo }}$ was determined as if it were estimated from expressed CES-1 studies. Then the enzyme ontogeny data for CES-1 was used to estimate the age-specific total hepatic metabolic clearance in vivo for deltamethrin. CES-1 shows a rapid maturation in humans and reaches the adult

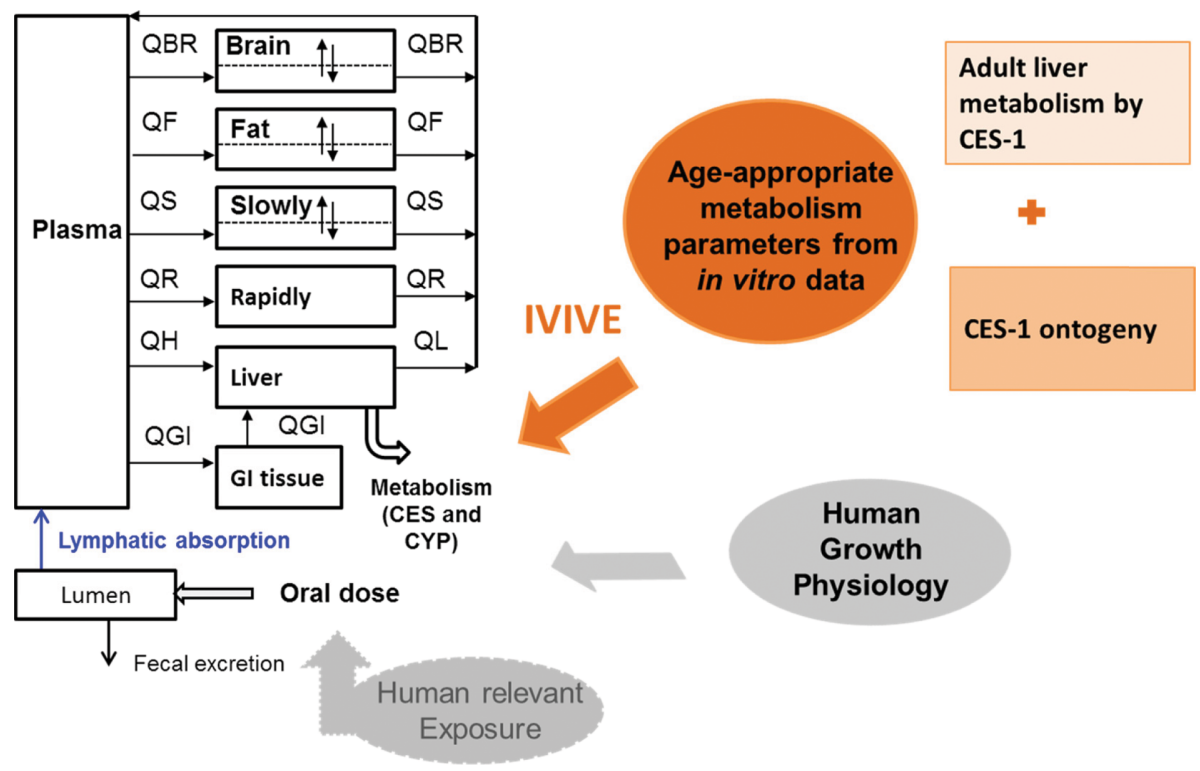

Fig. 3. Developing a human PBPK model for deltamethrin for different ages. 
level within 3 months after birth (44). Human growth physiology (7) has been integrated along with these in vitro-estimated age-appropriate metabolic constants as illustrated in Fig. 3 into the PBPK model. Age-specific exposure levels will be coherently integrated into the same PBPK modeling platform in the future.

Given that our purpose is to demonstrate the IVIVE process and show the expected outcomes of age-specific internal exposure at the target, we only used the average Clint ${ }_{\text {in vivo }}$ for each age instead of simulating its distribution in a population in the current preliminary modeling. In the PBPK

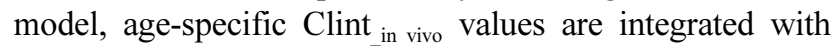
other age-dependent parameters such as liver weight, hepatic blood flow and additional IVIVE scaling factors (e.g., microsomal or cytosolic protein per g liver). For deltamethrin, the intrinsic metabolic clearance of deltamethrin in the liver in early ages was higher than that in adults (40). The resulting maximum brain concentrations (Cmax) after a single exposure across ages showed a slight increase with age. In contrast, the animal studies exhibited decreased metabolic clearance and increased brain concentrations (40). This was explained by efficient hydrolysis by CES-1 as well as its early maturation in expression in the liver during human development. The model predicted brain concentrations at different ages can be used to compare internal exposures among different ages and to compare in vitro or in vivo neurotoxicity studies to support safety assessments for pyrethroids for early ages.

\section{CONCLUSION}

The ultimate goal of the IVIVE-PBPK modeling presented here is to improve risk assessment for sensitive populations. With the pyrethroid case study presented here, we demonstrate the validity and utility of the PBPK models parameterized with in vitro metabolism data in predicting target tissue and plasma concentrations of a chemical in different ages of humans under various exposure conditions. The ability to simulate realistic exposure condition is dependent on the capability of PBPK models to connect to and use the outputs from exposure models such as CARES and SHEDS. The model-predicted target tissue or plasma concentrations or other dose-metrics can be used to develop chemical-specific adjustment factors for early age subpopulations in risk and safety assessment. Ultimately, they can be used to in conjunction with in vitro concentration-effect relationships to inform margins of safety for a given chemical exposure to support determining safe exposure guidelines in potentially sensitive populations for environmental chemicals.

\section{ACKNOWLEDGEMENT}

This research is funded by the Council for Advancement of Pyrethroid Human Risk Assessment (CAPHRA).

\section{REFERENCES}

1. Andersen, M.E. and Krewski, D. (2010) The vision of toxicity testing in the 21 st century: moving from discussion to action. Toxicol. Sci., 117, 17-24.

2. Judson, R.S., Kavlock, R.J., Setzer, R.W., Hubal, E.A., Martin, M.T., Knudsen, T.B., Houck, K.A., Thomas, R.S., Wetmore, B.A. and Dix, D.J. (2011) Estimating toxicity-related biological pathway altering doses for high-throughput chemical risk assessment. Chem. Res. Toxicol., 24, 451-462.

3. Knudsen, T.B., Houck, K.A., Sipes, N.S., Singh, A.V., Judson, R.S., Martin, M.T., Weissman, A., Kleinstreuer, N.C., Mortensen, H.M., Reif, D.M., Rabinowitz, J.R., Setzer, R.W., Richard, A.M., Dix, D.J. and Kavlock, R.J. (2011) Activity profiles of 309 ToxCast chemicals evaluated across 292 biochemical targets. Toxicology, 282, 1-15.

4. Ankley, G.T., Bennett, R.S., Erickson, R.J., Hoff, D.J., Hornung, M.W., Johnson, R.D., Mount, D.R., Nichols, J.W., Russom, C.L., Schmieder, P.K., Serrrano, J.A., Tietge, J.E. and Villeneuve, D.L. (2010) Adverse outcome pathways: a conceptual framework to support ecotoxicology research and risk assessment. Environ. Toxicol. Chem., 29, 730-741.

5. Clewell, H.J., Tan, Y.M., Campbell, J.L. and Andersen, M.E. (2008) Quantitative interpretation of human biomonitoring data. Toxicol. Appl. Pharmacol., 231, 122-133.

6. Yoon, M., Cambell, J.L., Andersen, M.E. and Clewell, H.J. (2012) Quantitative in vitro to in vivo extrapolation of cellbased toxicity assay results. Crit. Rev. Toxicol., 42, 633-652.

7. Clewell, H.J., Gentry, P.R., Covington, T.R., Sarangapani, R. and Teeguarden, J.G. (2004) Evaluation of the potential impact of age- and gender-specific pharmacokinetic differences on tissue dosimetry. Toxicol. Sci., 79, 381-393.

8. Saghir, S.A., Khan, S.A. and McCoy, A.T. (2012) Ontogeny of mammalian metabolizing enzymes in humans and animals used in toxicological studies. Crit. Rev. Toxicol., 42, 323-357.

9. Clewell, H.J., Teequarden, J., McBonald, T., Sarangapani, R., Lawrence, G., Covington, T., Gentry R. and Shipp, A. (2002) Review and evaluation of the potential impact of age- and gender-specific pharmacokinetic differences on tissue dosimetry. Crit. Rev. Toxicol., 32, 329-389.

10. Clewell, H.J., Reddy, M.B., Lave, T. and Andersen, M.E. (2007) Physiologically Based Pharmacokinetic Modeling. Preclinical Development Handbook, John Wiley and Sons, Inc. pp. 1167-1227.

11. Phillips, M.B., Yoon, M.Y., Young, B. and Tan, Y.M. (2014) Analysis of biomarker utility using a PBPK/PD model for carbaryl. Front. Pharmacol., 5, 246.

12. Price, P.S., Schnelle, K.D., Cleveland, C.B., Bartels, M.J., Hinderliter, P.M., Timchalk, C. and Poet, T.S. (2011) Application of a source-to-outcome model for the assessment of health impacts from dietary exposures to insecticide residues. Regul. Toxicol. Pharmacol., 61, 23-31.

13. Brown, R.P., Delp, M.D., Lindstedt, S.L., Rhomberg, L.R. and Beliles, R.P. (1997) Physiological parameter values for physiologically based pharmacokinetic models. Toxicol. Ind. Health, 13, 407-484.

14. Wu, H., Yoon, M.Y., Verner, M.A., Xue, J., Luo, M., Andersen, M.E., Longnecker, M.P. and Clewell, H.J. (2015) Can the observed association between serum perfluoroalkyl sub- 
stances and delayed menarche be explained on the basis of puberty-related changes in physiology and pharmacokinetics? Environ. Int., 82, 61-68.

15. Price, P.S., Conolly, R.B., Chaisson, C.F., Gross, E.A., Young, J.S., Mathis, E.T. and Tedder, D.R. (2003) Modeling interindividual variation in physiological factors used in PBPK models of humans. Crit. Rev. Toxicol., 33, 469-503.

16. Houston, J.B. and Galetin, A. (2008) Methods for predicting in vivo pharmacokinetics using data from in vitro assays. Curr. Drug Metab., 9, 940-951.

17. Jamei, M., Marciniak, S., Edwards, D., Wragg, K., Feng, K., Barnett, A. and Rostami-Hodjegan, A. (2013) The simcyp population based simulator: architecture, implementation, and quality assurance. Silico Pharmacol., 1, 9.

18. Jamei, M., Marciniak, S., Feng, K., Barnett, A., Tucker, G. and Rostami-Hodjegan, A. (2009) The Simcyp population-based ADME simulator Expert Opin. Drug Metab. Toxicol., 5, 211-223.

19. Rostami-Hodjegan, A. and Tucker, G.T. (2007) Simulation and prediction of in vivo drug metabolism in human populations from in vitro data. Nat. Rev. Drug Discovery, 6, 140-148.

20. Clewell, H.J. and Andersen, M.E. (1996) Use of physiologically based pharmacokinetic modeling to investigate individual versus population risk. Toxicology, 111, 315-329.

21. Clewell, H.J. (1993) Coupling of computer modeling with in vitro methodologies to reduce animal usage in toxicity testing. Toxicol. Lett., 68, 101-117.

22. Hinderliter, P.M., Price, P.S., Bartels, M.J., Timchalk, C. and Poet, T.S. (2011) Development of a source-to-outcome model for dietary exposures to insecticide residues: an example using chlorpyrifos. Regul. Toxicol. Pharmacol., 61, 82-92.

23. Kedderis, G.L. (2007) In vitro to in vivo extrapolation of metabolic rate constants for physiologically based pharmacokinetic models. Toxicokinet. Risk Assess., 185-210.

24. Yoon, M., Kedderis, G.L., Yan, G.Z. and Glewell, H.J. (2015) Use of in vitro data in developing a physiologically based pharmacokinetic model: Carbaryl as a case study. Toxicology, 332, 52-66.

25. Groothuis, F.A., Heringa, M.B., Nicol, B., Hermens, J.L., Blaauboer, B.J. and Kramer, N.I. (2015) Dose metric considerations in in vitro assays to improve quantitative in vitro-in vivo dose extrapolations. Toxicology, 332, 30-40.

26. Johnson, T.N., Rostami-Hodjegan, A. and Tucker, G.T. (2006) Prediction of the clearance of eleven drugs and associated variability in neonates, infants and children. Clin. pharmacokinet., 45, 931-956.

27. Nong, A., McCarver, D.G., Hines, R.N. and Krishnan, K. (2006) Modeling interchild differences in pharmacokinetics on the basis of subject-specific data on physiology and hepatic CYP2E1 levels: a case study with toluene. Toxicol. Appl. Pharmacol., 214, 78-87.

28. Wetmore, B.A., Allen, B., Clewell, H.J., Parker, T., Wambaugh, J.F., Almond, L.M. and Thomas, R.S. (2014) Incorporating population variability and susceptible subpopulations into dosimetry for high-throughput toxicity testing. Toxicol. Sci., 142, 210-224.

29. Hines, R.N. (2008) The ontogeny of drug metabolism enzymes and implications for adverse drug events. Pharmacol. Ther., 118, 250-267.

30. Rowland, M., Peck, C. and Tucker, G. (2011) Physiologically- based pharmacokinetics in drug development and regulatory science. Annu. Rev. Pharmacol. Toxicol., 51, 45-73.

31. Sheets, L.P., Doherty, J.D., Law, M.W., Reiter, L.W. and Crofton, K.M. (1994) Age-dependent differences in the susceptibility of rats to deltamethrin. Toxicol. Appl. Pharmacol., 126, 186-190.

32. Anand, S.S., Kim, K.B., Padilla, S., Muralidhara, S., Kim, H.J., Fisher, J.W. and Bruckner, J.V. (2006) Ontogeny of hepatic and plasma metabolism of deltamethrin in vitro: role in age-dependent acute neurotoxicity. Drug Metab. Dispos., 34, 389-397.

33. Kim, K.B., Anand, S.S., Kim, H.J., White, C.A., Fisher, J.W., Tornero-Velez, R. and Bruckner, J.V. (2010) Age-, dose-and time-ependency of plasma and tissue distribution of deltamethrin in immature rats. Toxicol. Sci., 115, 354-368.

34. Crow, J.A., Borazjani, A., Potter, P.M. and Ross, M.K. (2007) Hydrolysis of pyrethroids by human and rat tissues: examination of intestinal, liver and serum carboxylesterases. Toxicol. Appl. Pharmacol., 221, 1-12.

35. Godin, S.J., Scollon, E.J., Hughes, M.F., Potter, P.M., DeVito, M.J. and Ross, M.K. (2006) Species differences in the in vitro metabolism of deltamethrin and esfenvalerate: differential oxidative and hydrolytic metabolism by humans and rats. Drug Metab. Dispos., 34, 1764-1771.

36. Hideo, K. (2012) Biotransformation and enzymes responsible for metabolism of pyrethroids in mammals, in parameters for pesticide QSAR and PBPK/PD models for human risk assessment. Am. Chem. Soc., 41-52.

37. Ross, M.K., Borazjani, A., Edwards, C.C. and Potter, P.M. (2006) Hydrolytic metabolism of pyrethroids by human and other mammalian carboxylesterases. Biochem. Pharmacol., 71, 657-669.

38. Hines, R.N. (2007) Ontogeny of human hepatic cytochromes P450. J. Biochem. Mol. Toxicol., 21, 169-175.

39. Yang, D., Pearce, R.E., Wang, X., Gaedigk, R., Wan, Y.J. and Yan, B. (2009) Human carboxylesterases HCE1 and HCE2: ontogenic expression, inter-individual variability and differential hydrolysis of oseltamivir, aspirin, deltamethrin and permethrin. Biochem. Pharmacol., 77, 238-247.

40. Clewell, H.J. and Yoon, M. (2015) Predicting pyrethroids target tissue exposure across ages: application of in vitro-in vivo extrapolation and physiologically based pharmacokinetic modeling. EPA-HQ-OPP-2015-0130.

41. Mirfazaelian, A., Kim, K.B., Anand, S.S., Kim, H.J., TorneroVelez, R., Bruckner, J.V. and Fisher, J.W. (2006) Development of a physiologically based pharmacokinetic model for deltamethrin in the adult male Sprague-Dawley rat. Toxicol. Sci., 93, 432-442.

42. Tornero-Velez, R., Mirfazaelian, A., Kim, K.B., Anand, S.S., Kim, H.J., Haines, W.T., Bruckner, J.V. and Fisher, J.W. (2010) Evaluation of deltamethrin kinetics and dosimetry in the maturing rat using a PBPK model. Toxicol. Appl Pharmacol., 244, 208-217.

43. Lake, B.G. (2015) Studies on the in vitro metabolism of deltamethrin by rat and human tissue preparations. EPA-HQOPP-2015-0130.

44. Pope, C.N., Karanth, S., Liu, J. and Yan, B. (2005) Comparative carboxylesterase activities in infant and adult liver and their in vitro sensitivity to chlorpyrifos oxon. Regul. Toxicol. Pharmacol., 42, 64-69. 